\title{
Una perspectiva antropológica de las organizaciones desde la caracterización sociocultural, administración y contabilidad social. Caso institución de educación superior*
}

doi:10.11144/Javeriana.cc17-43.paoc

\section{Carmen Luz Cely-López}

Antropóloga, Universidad Nacional de Colombia, Bogotá. Magíster en evaluación de la educación, Universidad Santo Tomás, Bucaramanga, Colombia. Especialista en teoría, métodos y técnicas de investigación social, Universidad Industrial de Santander, UIS, Bucaramanga. Especialista en docencia universitaria, Universidad Autónoma de Bucaramanga, UNAB. Docente tiempo completo, Universidad Cooperativa de Colombia.

Correo electrónico: carmen.cely@campusucc.edu.co

\begin{abstract}
Ofelia Gómez-Niño
Contadora pública, Universidad Autónoma de Bucaramanga. Especialista en docencia universitaria, Universidad Cooperativa de Colombia. Magíster en administración, Universidad Santo Tomás y candidata a doctora, Universidad Nacional de Rosario, UNR, Argentina. Docente de medio tiempo, Universidad Cooperativa de Colombia, sede Bucaramanga. Profesora de consultorio contable I-II y escuelas del pensamiento contable.
\end{abstract}

Correo electrónico: Ofelia.gomez@campusucc.edu.co

\footnotetext{
* Este es un artículo de investigación. El contenido del documento se deriva de los resultados del proyecto denominado Relaciones culturales, interculturales y socioeducativas en el contexto organizacional. Caso una institución de educación superior, en la ciudad de Bucaramanga. El objetivo general del trabajo consiste en identificar las relaciones culturales, interculturales y socioeducativas que se favorecen en el contexto de las organizaciones y los objetivos específicos se orientan a determinar las relaciones interculturales que favorecen la dinámica organizacional e interpretar las relaciones socioeducativas que se generan entre la fuerza laboral de la institución educativa objeto de estudio. El desarrollo del trabajo se realizó con la aprobación de fondos del Comité Nacional para el Desarrollo de la Investigación, CONADI, Universidad Cooperativa de Colombia.
} 
Resumen Este estudio se enfocó en identificar desde la antropología cultural las relaciones culturales e interculturales y socioeducativas que se establecen en las organizaciones y su incidencia en el clima laboral para el logro de los propósitos misionales, desde la perspectiva de la administración y la contabilidad social. Se estudió una institución de educación superior, perteneciente al sector de la economía solidaria; el alcance fue el conocimiento del tejido social de las organizaciones para mostrar cómo desde la comprensión de las relaciones y los significados que se establecen en la organización pueden llegar a mayor productividad. Los indicadores cualitativos permiten evaluar ventajas comparativas de la gestión del talento humano en función del conocimiento, los aprendizajes y el compromiso con los propósitos misionales u organizacionales. Los instrumentos para recolección de información fueron entrevistas semiestructuradas, grupos focales y observación participante. Las categorías emergentes son relaciones laborales, cultura e interculturalidad, aspectos socioeducativos y adaptación.

Palabras claves Administración; antropología organizacional; contabilidad social; cultura; cultura organizacional; interculturalidad; relaciones laborales

\section{Códigos JEL M00}

\section{An Anthropological Perspective of Organizations from Sociocultural Characterization, Management, and Social Accounting. The Case of a Tertiary Education Institution}

\footnotetext{
Abstract This study focused on identifying from cultural anthropology studies, cultural and intercultural relations and socio-educational established in organizations and the impact on the working environment for the achievement of the mission purposes, from the perspective of management and social accounting. An institution of higher education, belonging to the solidarity economy sec-
}

tor was studied; the scope was aimed to knowledge of the social fabric of organizations to show how starting from the understanding of relationships and meanings set forth in the organization can reach higher productivity.

The qualitative indicators let to assess comparative advantages of human talent management in terms of knowledge, learning and commitment to the mission or organizational purposes. The instruments for data collection were semistructured interviews, focus groups and participant observation. The emergent categories are labor relations, culture and inter-cultures, social educative aspects and adaptation.

Keywords Management; organizational anthropology; social accounting; culture; interculturality; working relations; productivity

\section{Perspectiva antropológica das organizações desde a caracterização sociocultural, administração e contabilidade social. Caso instituição de ensino superior}

Resumo Este estudo centrou-se na identificação da antropologia cultural e relações culturais e sócio-culturais estabelecidos nas organizações e seu impacto sobre o ambiente de trabalho para a realização dos objectivos da missão, a partir da perspectiva da administração social e contabilidade. uma instituição de ensino superior, pertencente ao sector da economia solidária estudada; o escopo era o conhecimento do tecido social das organizações para mostrar como de compreender as relações e significados estabelecidos na organização pode alcançar maior produtividade. Os indicadores qualitativos para avaliar as vantagens comparativas da gestão de talento humano em termos de conhecimento, aprendizagem e compromisso com os propósitos organizacionais missão ou. Os instrumentos de coleta de dados foram entrevistas semi-estruturadas, grupos focais e observação participante. Categorias emergentes são relações de trabalho, a cultura e intercultural, aspectos sociais e educacionais e adaptação. 
Palavras-chave administração; antropologia organizacional; contabilidade social; cultura; cultura organizacional; multiculturalismo; relações de trabalho

\section{Introducción}

Si en el mundo de hoy un antropólogo no entiende de ingeniería, economía y empresa, es como si no entendiera de relaciones de parentesco y cultura.

Davydd Greenwood

Las organizaciones son comunidades sociales en las que se establecen una serie de relaciones laborales, económicas, profesionales, culturales y afectivas para la satisfacción de necesidades e intereses comunes. De acuerdo con la apreciación anterior, se toma como referente lo expuesto por Susan Wright (2005, citada por Juan Pablo Gonnet, 2012, p. 9), que "propone que por cultura organizacional se debe entender, de acuerdo a la concepción semiótica de cultura sostenida por un sistema de redes de significado a partir de las cuales los actores interpretan sus propias prácticas negociando significados". La antropología ha incursionado en todas las actividades que el hombre realiza, entre ellas la vida laboral, eje determinante en una organización para comprender los comportamientos, costumbres, creencias, normas, valores, estilos de vida, nivel educativo de las personas.

Además, tiene en cuenta la diversidad desde la interculturalidad como un hecho fundamental en que se mueven los seres humanos en la globalización para aceptar la diferencia; el control interno y externo en una organización genera los ambientes para una buena adminis- tración. "La cultura como mecanismo de control social es importante porque ofrece varias ventajas sobre el control externo logrado a través de premios y sanciones" (Pfeffer, 2000, p. 168). La antropología da el aporte de cultura, interculturalidad y el papel de esta disciplina en las organizaciones; la administración como proceso de organización, dirección y control; la contabilidad social que se ocupa de aspectos relacionados con empleados, comunidad social, factores del medio ambiente y cuestiones éticas de la organización desde la responsabilidad social.

Aunque todas las sociedades modernas comparten muchos intereses comunes, sería un error desechar las diferencias culturales e intranacionales por considerarlas poco importantes. El curso de la historia ha forzado muchas modificaciones en las características sociales nacionales en los puntos de vista sobre el significado de la vida, en los estilos y filosofía de la organización y de la dirección (Morgan, 1998, p. 100).

Las disciplinas que direccionan el artículo desde la antropología sociocultural, administración y contabilidad social han incursionado al estudio de las organizaciones de manera reciente desde la mirada cultural, el aporte que de aquí subyace ayudará a destacar el valor de las relaciones en el contexto de las organizaciones empresariales, a la resolución de problemas y contribuirá a ampliar elementos teóricos de la antropología organizacional, para comprender la realidad y las relaciones que se establecen en las organizaciones ${ }^{1}$.

1 Las autoras agradecen a la Universidad Cooperativa de Colombia, Sede Bucaramanga, institución objeto de estu- 


\section{Metodología, material y métodos}

La presente investigación se realiza desde el paradigma cualitativo descriptivo e interpretativo de carácter etnográfico, parafraseando a Rosana Guber (2000), como método y como enfoque para comprender desde el punto de vista de los actores las caracterizaciones socioculturales de la organización y las relaciones que se entretejen en ella, que permita la interpretación de los hechos. Porque hacer etnografía es - como dice Clifford Geertz (1992) — "establecer relaciones, seleccionar informantes, transcribir textos, establecer genealogías, trazar mapas de área llevar un diario... es como tratar de leer (en el sentido de "interpretar un texto")". Lo particular de este tipo de investigación es la simultaneidad de los procesos que vuelven realidad los elementos teóricos descritos y los datos que constituyen la evidencia del tipo de relaciones planteadas.

Este tipo de investigación centra la atención en la descripción, interpretación y comprensión de los aspectos culturales, como lo expresa Miguel Martínez-Miguélez (2000): “intenta hacer una aproximación global de las situaciones sociales para explorarlas, descubrirlas y comprenderlas de manera inductiva”, al conocer e identificar las relaciones culturales, interculturales y socioeducativas que favorecen el contexto organizacional desde una visión integral, dando las propias significaciones e interpretaciones de acuerdo con la información

dio, a los grupos participantes y agentes que de una u otra forma contribuyeron para que esta investigación cumpliera los propósitos trazados. recogida en el trabajo de campo. Dice Rosana Guber (2000) al respecto: "Una buena interpretación es aquella que no los malinterpreta". En consecuencia, se ratifica que la etnografía es una herramienta fundamental que facilita el análisis de una organización; mirada así, capta la realidad social, ayuda a revelar relaciones laborales de las personas que allí trabajan y que vienen de diferentes contextos sociales y culturales.

Para captar cómo conciben los individuos la realidad en las organizaciones en la interacción constante del desempeño laboral se hace una mirada interpretativa desde de la contabilidad social a partir de los planteamientos de Wai Fong Chua (como la citó Hugo A. Macías, 2013).

En esencia, la alternativa interpretativa busca darles sentido a las acciones humanas... se asume que en el mundo social cada acción humana tiene un propósito, tiene un mundo de significados predeterminados, está arraigada en experiencias pasadas; para estudiar los actores en su mundo cotidiano y comprender las prácticas, se acude al trabajo etnográfico, el estudio de caso y la observación participante... relación cercana entre teoría y práctica, ya que revela qué hacen las personas mientras interactúan y hablan; su objetivo es comprender las acciones en sus entornos naturales. En consecuencia, la investigación interpretativa no tiene asunciones a priori, su papel es emergente y simbólico, está enraizado en los procesos sociales; los actores definen cada una de las categorías y no se buscan explicaciones ge- 
neralizables (Macías, 2013, p. 703, que cita a

Wai Fong Chua, 1986).

\section{Caracterización del grupo. Esta investiga-} ción es un estudio de caso en una institución de educación superior de la ciudad de Bucaramanga interesada en dinamizar los procesos a la luz de la antropología y las relaciones culturales, interculturales y socioeducativas que se desarrollan en el contexto empresarial con el aporte de las ciencias administrativas y la contabilidad social. Para tal fin, se hizo un "mapeo", para lograr un acercamiento al objeto de estudio. Se tomaron las diferentes personas que laboran en la universidad, se clasifican directivos, docentes; personal cualificado y no cualificado (secretarias y de servicios generales), hasta cuando hubo saturación de la información. Este proceso está sujeto a la dinámica de la recolección de información derivada de los mismos hallazgos.

Técnicas de recolección de información. Los instrumentos utilizados se enuncian a continuación:

- Entrevistas semiestructuradas, dirigidas al personal directivo, docente y administrativo de la organización. El objetivo es hacer que hablen sobre lo que saben, piensan y creen que significan las relaciones que se establecen en el contexto de la organización, desde lo cultural, intercultural y socioeducativo.

- Observación participante con el fin de percibir situaciones acerca de lo que hacen y dicen en la cotidianidad laboral las personas que suministran información; se utiliza guía para el registro del interactuar diario.
- Charlas informales orientadas a conocer las percepciones e interpretaciones acerca de la institución.

- Grupos focales conformados por mujeres, uno integrado por las empleadas de servicios generales y el otro por las secretarias de las diversas dependencias; esto con el fin de contrastar la información sobre la percepción de las diferentes interacciones en la vida laboral.

- Clasificación de la información por categorías de análisis teórica y emergente, como relación laboral, entorno sociocultural, interculturalidad y adaptación y la conformación de las dimensiones y subdimensiones según los instrumentos aplicados.

El análisis de la información se realiza según imaginarios relevantes de cada uno de los grupos participantes; se compararon e interpretaron a la luz de los elementos teóricos del interaccionismo simbólico y el aporte de la antropología en las organizaciones y la mirada de la administración y la contabilidad social para discutir resultados. Se triangula la información de entrevistas, grupos focales, observación y charlas informales con el personal de la institución y se sustenta en la teoría que fundamenta la investigación.

La investigación tiene un enfoque comprensivo como lo expone Carlos A. SandovalCasilimas (1996). La captación — mediante la interpretación y el diálogo- del sentido de lo que el otro o los otros quieren decir con otras palabras o silencios, con las acciones o las inmovilidades, sino también la posibilidad de construir generalizaciones que permitan enten- 
der los aspectos comunes a muchas personas y grupos humanos en su proceso de producción y apropiación de la realidad social y cultural en que desarrollan la existencia. Las categorías de análisis son relaciones laborales, cultura e interculturalidad, aspectos socioeducativos y adaptación; se analizan según expresiones de los participantes agrupados por elementos comunes; el personal entrevistado corresponde a cada uno de los grupos observados.

\section{Referente teórico}

La realidad y la comprensión de las acciones de los individuos que interactúan en una organización son variadas, las perspectivas han cambiado desde la gestión humana por la influencia de la sociedad de la información y del conocimiento, porque las personas aprenden y transfieren el aprendizaje a las organizaciones. A la luz del trabajo etnográfico, el valor de la antropología sociocultural en el contexto de las organizaciones empresariales apoya la resolución de problemas en ellas y contribuye con elementos teóricos de la antropología organizacional, para comprender comportamientos y relaciones que se establecen en las instituciones.

La antropología busca entender, comprender y explicar la realidad social y captar el carácter normal sin reducir a particularidades, mirando objetos de la vida social de manera integral y subjetiva. "La antropología explora la diversidad humana en el tiempo y el espacio; estudia la condición humana, su pasado, presente y futuro, su biología, sociedad, el lenguaje y la cultura. De particular interés es la diversidad que proviene de la adaptabilidad huma- na" (Kottak, 2011). Mientras la antropología sociocultural centra la mirada en las caracterizaciones de las sociedades. En el caso de la antropología aplicada, el interés es buscar soluciones a problemas mediante la investigación y generar nuevos conocimientos que permitan explicar las problemáticas sociales encontradas en ese proceso de intervención; este campo de acción enfoca también el horizonte de estudio hacia las organizaciones y la vida empresarial.

La cultura es un concepto fuerte de la antropología y es la manera como una sociedad demarca el estilo de vida y se caracteriza de acuerdo a ella. La cultura hace referencia a conductas, actitudes y pensamientos del hombre aprendidos dentro de un grupo social o más exactamente en la sociedad en que se ha desarrollado. Los propósitos expresados por Clifford Geertz (1992, 2003 p. 27) son fundamentales, "al observar la cultura no como una entidad, sino como 'algo' a lo que puedan atribuirse de manera casual acontecimientos sociales, modos de conducta; la cultura es un contexto dentro del cual pueden describirse todos los fenómenos de manera inteligible, es decir "densa". Comprender la cultura de un pueblo supone captar el carácter normal sin reducir la particularidad”. La cultura implica entenderla como un proceso de comprensión permanente entre los miembros de las organizaciones. Los planteamientos anteriores identifican elementos expuestos por Susan Wright (2005, como la citó Juan Pablo Gonnet, 2012) que propone: "la cultura organizacional se debe entender de acuerdo a la concepción semiótica de cultura sostenida por un sistema de redes de significa- 
do, a partir de las cuales los actores interpretan sus propias prácticas negociando significados" (Gonnet, 2012).

Así mismo, la contabilidad social en la empresa se ocupa de aspectos relacionados con empleados, comunidad social, factores del medio ambiente y cuestiones éticas de la organización, desde la responsabilidad social. Al observar la realidad y la comprensión de las acciones de los individuos que interactúan en una organización son variadas, las perspectivas de la gestión humana han cambiado por la influencia de la sociedad de la información y del conocimiento. La contabilidad social es un subsistema que identifica y mide "los efectos económicos y sociales de la empresa sobre la sociedad pero también los efectos de la sociedad sobre la empresa" (Rey, 1978, p. 34); estos efectos son medidos por la responsabilidad social, el balance social y la contabilidad del talento humano, el último es gestor de la productividad y eficiencia de los procesos orientados al logro de los objetivos empresariales (Jiménez-Aguirre, 2008).

Las organizaciones son entes sociales que mantienen una estructura sistémica como un conjunto de redes que establece la relación con agentes internos y externos, esto se refiere a los stakeholders; en estas relaciones está presente la teoría contable, fundamentada en la contabilidad social.

La contabilidad que refleja la realidad a través de una serie de atributos, variables o cuentas, las cuales se plasman en una serie de informes que contienen un lenguaje específico. Es posible pensar en estos atributos, según el nivel de complejidad que se alcance mediante la implementación de un modelo contable, el cual dependerá de la complejidad de la teoría que se utilice (Machado-Rivera, 2007, p. 70).

En la actualidad, la contabilidad se desenvuelve en una sociedad que busca la maximización del capital, vale decir, el financiero. Así, los avances que se van gestando en lo contable buscan propiciar las herramientas y los instrumentos para el alcance de este fin.

El profesor Carlos Mario Ospina-Zapata propone que la contabilidad expresa una representación de hechos socioeconómicos que subyacen a una organización y sus relaciones intercontextuales. Que tal representación supone una lógica de comprensión de la realidad, un tipo de racionalidad, que funciona como cálculo de la evolución del capital con elementos relevantes como la renta y la apropiación. Así, la contabilidad debe avanzar en nuevas dinámicas que permitan la representación de una realidad social, no solo económica, pues la contabilidad es un potente sistema para entender-comprender-intervenir la realidad socioeconómica (Ospina-Zapata, 2006).

Es importante mencionar que la contabilidad tiene una función de apoyo a todo el sistema organizacional, es el eje que sustenta toda la información económica, financiera, administrativa, social y del recurso humano. "El capital humano es inalienable. Es físicamente imposible separar el acervo de capital humano de su poseedor, ... no puede haber ningún mercado para este recurso en su forma capitalizada. Los contratos a largo plazo para los flujos de capital humano no se pueden hacer cumplir legalmente por el comprador en muchas 
sociedades" (Sunder, 2005, p. 67). Desde este planteamiento, la contabilidad social se transversaliza en todas las áreas de la entidad y busca el equilibrio de las diversas interpretaciones y significaciones que esta tiene.

Se puede agregar otra mirada de las organizaciones a partir de lo administrativo o gerencial. Como lo expresa Susan Wright (citada por Juan Pablo Gonnet, 2012, p. 9), hay dos perspectivas distintas de la cultura organizacional; por un lado, una visión "gerencialista" y, por otro lado, una visión "socioantropológica”. Este trabajo ha escudriñado, desde las dos perspectivas, con énfasis en la segunda a partir de los aportes del interaccionismo simbólico. Para mostrar la importancia de las organizaciones, partiendo del hecho de que la conformación la hacen los individuos, como menciona Edgar H. Schein (1988, citado por Gonnet, 2012, p. 4).

\footnotetext{
Parte de la premisa de que las organizaciones tienen culturas propias y desde aquí construye su teorización. En un nivel general, se entiende a la cultura organizacional como aquellas dimensiones inconscientes que afectan el modo en que los miembros de una institución piensan, sienten y actúan.
}

Ahora bien, parafraseando a Lydia Aurora Arbaiza-Fermini (2011), mirados desde la administración empresarial, los elementos de interculturalidad están presentes; puede decirse que no hay ni un solo modelo de funcionamiento, ni ambiente económico y social que pueda quedarse aislado del resto del mundo. Desde esta perspectiva, es posible afirmar que la administración intercultural se define como el estudio de la conducta organizacional de países y culturas; esta facilita la comprensión de las interacciones de los trabajadores, clientes, proveedores y socios para ampliar la visión en sí misma y así lograr un alcance de la administración multicultural.

En este orden de ideas, las instituciones deben poner total atención en definir códigos culturales que las caractericen, de modo que los trabajadores también se apropien y asuman estos como pilares de identidad con principios y valores que se profesan dentro y fuera de la institución, como imagen corporativa. Lo anterior se sustenta también con los comentarios de estudios organizacionales que han tendido a presentar la cultura como una dimensión reificada. Esto significa que se ha definido a la cultura como una "variable" que afecta externamente o internamente a la organización, en un sentido más o menos positivo o negativo para sus dos finalidades: el sistema de gestión y control organizacional (Gonnet, 2012, p. 8). De igual modo, se tuvo en cuenta la percepción de Felipe Cáceres-Rubio y Mónica VillacrésChaparro (2010), sobre cultura organizacional, debido a que surge de la agrupación de seres humanos, que persigue una finalidad específica, e implica una organización sociocultural, en la que se establecen normas y valores que orienten la conducta colectiva y ahí, los individuos tejen una serie de imaginarios sociales.

Antropológicamente, el imaginario es la percepción, las creencias que los individuos tienen y tejen para construir y comprender la realidad como un proceso de construcción social generado por la interacción humana, para posibilitar de esa manera el reconocimiento 
del mundo simbólico creado por el hombre en las sociedades y va muchas veces más allá de la realidad. La realidad se construye mediante las diferentes miradas, opiniones y formas de captar la diferencia. Cornelius Castoriadis (1975, 1998) dice que la historia de la humanidad es la historia del imaginario humano y de sus obras. Las significaciones son un conjunto de valores, de representaciones que tienen sentido para los individuos en una sociedad (Castoriadis, 1975, 1998). En el contexto institucional, los individuos hacen las propias interpretaciones de lo que allí acontece.

Las instituciones y las significaciones imaginarias sociales deben ser coherentes. La coherencia tiene que ser estimada desde un punto de vista inmanente, es decir, en relación a las características y a los principales "impulsos" de la sociedad considerada; teniendo en cuenta el comportamiento conforme a los individuos socializados... Por otra parte, las instituciones y las significaciones imaginarias sociales deben ser completas (Castoriadis, 1997).

\section{Resultados y discusión}

La eficiencia y calidad en los procesos organizacionales son posibles solo si se logra instituir liderazgo, ayudando al personal a trabajar mejor. William Edwards Deming

Los grupos objeto de estudio están integrados por el personal que labora en la organización; participaron directivos, administrativos, docentes y otros (secretarias, servicios generales). Estos funcionarios se caracterizan por tener diversos niveles de formación académica, desde bachillerato incompleto, técnicos, tecnólogos, profesionales, especialistas y maestría. Hay mayor participación de la mujer en cargos directivos, administrativos, docentes y otros funcionarios (gráfico 1).

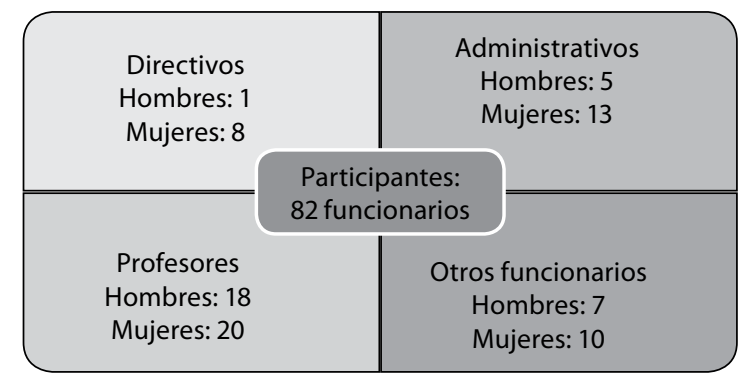

Gráfico 1

Población según género y desempeño laboral

Fuente: elaboración propia, a partir de la información del trabajo de campo 
Los participantes se clasifican en cuatro grupos: directivos, administrativos, docentes y otros funcionarios (secretarias, servicios generales). Se elaboran las categorías teóricas analíticas y las emergentes surgidas de los mismos resultados; se demarcan las dimensiones y subdimensiones. El encuadre para describir y explicar las realidades socioculturales en las relaciones laborales, como la interculturalidad y el proceso de adaptación de las personas que viven en la organización, fueron factores observados, abordados e interpretados a la luz de los planteamientos teóricos de la antropología, el interaccionismo simbólico y las teorías de la administración que tratan los ambientes laborales en el contexto organizacional, teniendo como base las categorías teóricas analíticas (tabla 1).

\begin{tabular}{lll}
\hline \multicolumn{1}{c}{ Categorías } & \multicolumn{1}{c}{ Dimensiones } & \multicolumn{1}{c}{ Subdimensiones } \\
\hline 1. Relaciones laborales & Compromiso laboral & Percepción \\
& Apoyo entre áreas funcionales & Manejo del entorno \\
& Relaciones con los compañeros & Actitud ante el trabajo \\
& Satisfacción laboral e institucional & Manejo del tiempo \\
& Comunicación & \\
2. Cultura e interculturalidad & Identidad colectiva & Aceptación del otro \\
& Tolerancia & Discriminación cultural \\
& Reconocimiento & Valores \\
& Respeto a la diferencia & Nivel educativo \\
3. Socioeducativa & Costumbres & \\
& Trabajo en equipo & Integración \\
& Cambio institucional & Cambio institucional \\
& Adaptación simbólica & Aculturación \\
& Adaptación espacial & Relaciones entre los grupos \\
& & Inconformidad institucional
\end{tabular}

Tabla 1

Marco de interpretación antropológica

Fuente: elaboración propia, a partir de información teórica y del trabajo de campo en general一, expresan que tienen y mantie-

Estas categorías salieron de la conformación teórica y la información emanada de las entrevistas semiestructuradas de los entrevistados y las dimensiones y subdimensiones correspondientes a las preguntas planteadas. Estas se analizan según expresiones de cada participante y se agrupan por elementos comunes; el personal entrevistado corresponde a cada uno de los grupos observados. Respecto a la primera categoría - relaciones laborales nen "sentido de pertenencia, compromiso y dedicación con las metas, compromiso con la institución. Siempre estoy de acuerdo y cumpliendo con responsabilidad y honestidad". También resaltan la importancia desde la "pertenencia y apoyo constante para el logro de los propósitos". Consideran que "el tiempo en la institución lo manejan cumpliendo las funciones de los cargos asignados". A la vez, ratifican que: "aprovechan el tiempo para planear, eje- 
cutar y darles prioridad a las metas de corto plazo". Presentan total compromiso con los objetivos institucionales.

Desde esta misma categoría, otros manifiestan: "estoy inconforme, circunstancia que lleva a que mi desempeño sea regular" y las relaciones "considero que son deficientes". "No hay espacios cómodos donde se pueda producir conocimiento e investigar". Al comparar estas evidencias, con relación a los aspectos comunicativos, los ven positivos sobre todo en lo que tiene qué ver con la percepción de "jefes, profesores y empleados es buena, porque las relaciones entre compañeros permiten el crecimiento personal, al igual que el trabajo por la construcción del conocimiento desde la experiencia”. Sin embargo, desde otro punto de vista, mencionan que "el apoyo interáreas es poco, existen demoras de tiempo y baja disposición para apoyar a otras áreas funcionales”. Reconocen que "a veces se trabaja de manera aislada y la comunicación no es oportuna y retrasa el cumplimiento de las metas". Perciben que la tecnología contribuye con la comunicación, pero los aleja la interacción personal: "la relación cara a cara se ha perdido". Pero también hacen hincapié: "cuando se necesita información de otras dependencias, no la suministran". "La información dada por los jefes a veces no es clara y se torna autoritaria e impositiva"; la comunicación tiene limitantes dentro de la organización (gráfico 2).

\section{Procesos comunicativos}

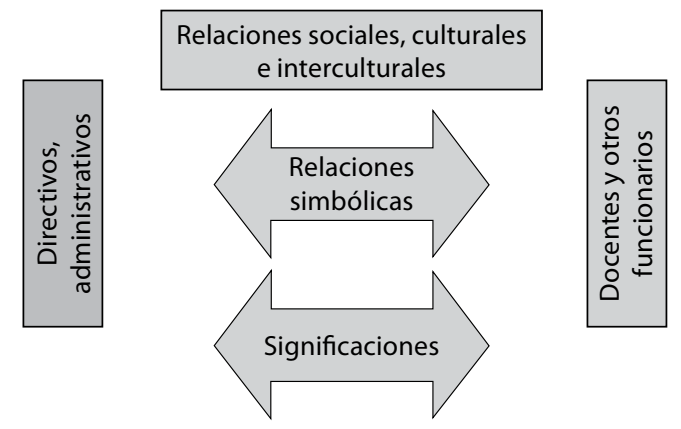

Gráfico 2

La comunicación: eje orientador de las relaciones empresariales

Fuente: elaboración propia con base en información teórica y trabajo de campo

Los participantes consideran que los procesos comunicativos en la organización son un factor que requiere reestructuración y mejoramiento, en lo concerniente a las relaciones sim- bólicas y las significaciones para que los canales de comunicación no distorsionen la información. Uno de los hechos es que "las reuniones se hacen a veces por teleconferencia por Skype 
y el calor humano se pierde". Consideran que el uso de medios tecnológicos es bueno; sin embargo, en exceso deshumaniza a la sociedad e incide en los ambientes laborales.

La comunicación define los medios para dar a conocer a las personas información que les permita direccionarse o encaminarse hacia los objetivos misionales y de este modo, crear unidades dinámicas en ambientes laborales acordes con las tendencias de las organizaciones modernas y abiertas. Los planteamientos del interaccionismo simbólico sustentan que "entre los humanos se producen los 'gestos significantes'. Estos requieren reflexión por parte del actor antes [de] que se produzca una reacción..." (Ritzer, 2002). En los procesos comunicativos, las personas interpretan las relaciones simbólicas y dan sus propias significaciones concernientes a las correlaciones que mantienen en su vida laboral (Chanlat, 1994). "El ser humano: un ser de palabra... gracias a esa facultad de expresar en palabras tanto la realidad de interior como la exterior podemos tener acceso al mundo de las significaciones".

En la segunda categoría, relaciones culturales e interculturales, se encontró que "percibo que hay respeto de diferencia de pensamiento. No existe discriminación por origen étnico, creencias, religión, política, condición social, opción sexual, género”. También lo ratifica otro grupo que indica "desde acá se reconocen los unos con los otros y no se da inicio de discriminación a pesar de las creencias, diversidad cultural y origen familiar, porque cada uno de nosotros venimos [sic] de diferentes lugares y hogares". Mencionan: "esto se debe a la naturaleza de la institución, donde prima el reconocimiento del otro, los valores cooperativos y la ayuda mutua". De otra parte, perciben que las "relaciones con los jefes han mejorado y por consiguiente estos los evalúan de manera adecuada".

De la misma manera, se observa que en algunas dependencias "las relaciones con los jefes no son las mejores, no hay respeto; por tanto, hay mal ambiente laboral y por consiguiente somos mal evaluados; se debe eliminar la subjetividad para este tipo de acciones...". "No me integro con nadie. No es bueno el trato con los compañeros. A veces, falta más compromiso. Hay compañeros que entorpecen el ambiente laboral". "La diversidad para unos afecta el ambiente organizacional; para otros, no la afecta", debido a que se adaptan fácilmente.

La interculturalidad es la relación entre dos o más culturas o grupos distintos que lleva a la aceptación de las diferencias; desde la visión antropológica (Gauthey, Ratiu, Rodgers \& Xardel, 1988, citados por Consuelo García de la Torre, 2007), la gestión intercultural en administración es aquella que tiene en cuenta en su concepción y en su práctica las diferencias entre culturas, desde su interrelación laboral y su aceptación dentro de la diversidad. Se aprecia, entonces, que los individuos definen la cultura institucional, a partir de las relaciones entre ellos y la organización. Lo demuestran con el sentido de cooperación, trabajo en equipo, responsabilidad y compromiso en el desarrollo de las actividades propias de los cargos. Este hecho hace que los resultados de las metas a cumplir sean acordes con la política de la institución con miras al cooperativismo y la cultura solidaria. 
La tercera categoría tiene qué ver con el entorno de los procesos socioeducativos, las diferencias sociales y económicas y de nivel educativo que caracterizan la discriminación a los compañeros de trabajo por este tipo de diferencias; se presentan aspectos significativos en los cuatro grupos de funcionarios, que expresaron: "me siento bien y no me he sentido discriminado"; "aceptable y significativa"; ese hecho de trabajar con personas con diferencias sociales, "esto hace que se fortalezca el nivel de tole- rancia". También indican que "no soy amigo de excluir a nadie, bajo ningún aspecto". A la vez, continuando con la aceptación, otros mencionan que "me siento bien, siempre y cuando cumplan con los compromisos". Los participantes concuerdan en que la filosofía institucional promueve la cultura del respeto por el otro, se reconocen los niveles jerárquicos en cuanto a líneas de autoridad y responsabilidad, se mantiene la relación de ayuda mutua y reconocimiento como iguales (gráfico 3).

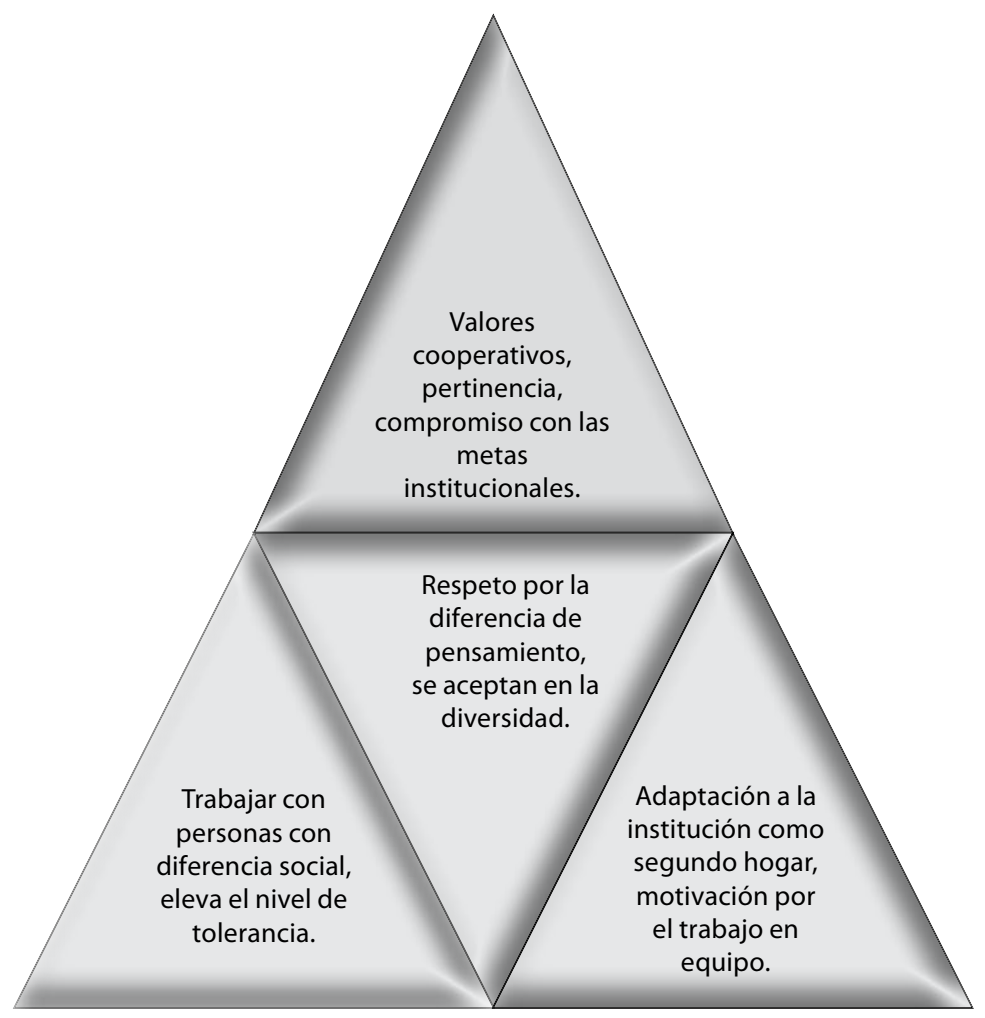

Gráfico 3

Factores dominantes en la gestión del talento humano

Fuente: elaboración propia, con base en la información de trabajo de campo 
La administración se esmera por hacer eficientes los recursos; en este caso, el capital humano que en gran medida es el motor del desarrollo de las empresas, organizaciones e instituciones. Las organizaciones existen gracias a la presencia de los humanos, porque estas son netamente sociales. Las personas valoran y reconocen las instituciones una vez deciden ser parte de ellas, hacen propios los valores, se comprometen con metas, se adaptan a las exigencias, reconocen a los compañeros y jefes a pesar de las diferencias; consideran que en la diversidad es posible aprender y crecer, se motivan con el trabajo en equipo por la creencia de que la unión hace la fuerza y buscan la sinergia como el medio para alcanzar la construcción colectiva y la integralidad.

La cuarta categoría es la adaptación. Para este grupo de trabajo se evidencia un alto grado de adaptabilidad y comentan: "mi institución es mi segundo hogar, porque permanecemos en ella la mayor parte del tiempo". "Me siento adaptado, pero no hay buenos espacios físicos para poder laborar de manera adecuada"; "la mejor forma de hacer crecer las empresas es mediante el trabajo en equipo y por eso, me siento bien". Perciben que trabajar en equipo permite obtener los resultados en mejores condiciones, las fortalezas de unos contribuyen a subsanar las debilidades de otros, ahora los logros de metas se miden por resultados conjuntos; creen que la unión hace la fuerza y que los que compiten por alcanzar los propósitos institucionales deben unir esfuerzos por medio de las competencias y experiencias para tener resultados óptimos y hacer aportes a los aprendizajes de la organización de forma significativa.
Hay percepciones negativas, por ejemplo: "No estoy adaptado y además no hay trato amable con las personas con las cuales nos relacionamos diariamente". "No escuchan a las personas y por tanto no hay buen ambiente". Sugieren a la institución cambios en el horario de lunes a viernes, es decir, cumplir las horas laborales en este lapso con el fin de no asistir el día sábado. De otra parte, mejorar el tipo de salario y en la contratación para mayor estabilidad laboral. Reestructurar la ubicación de las oficinas y medio de trabajo. Finalmente, se evidencia adaptación y estabilidad, debido a que los funcionarios tienen una antigüedad entre 2 y 17 años.

Integradas las cuatro categorías se resumen las percepciones de los agentes participantes con lo cual se definen factores dominantes: valores cooperativos, como filosofía institucional, pertinencia y compromiso para el logro de metas, respeto por la diferencia como medio de aceptación, interactuar con la diversidad social para elevar el nivel de tolerancia y de motivación por el trabajo en equipo para una construcción colectiva desde la perspectiva de la eficiencia. El trabajo con grupos focales recupera algunas percepciones antes no expuestas por los entrevistados, tanto de docentes como personal administrativo, que manifiestan desarraigo y poco interés con la institución, reducida aculturación y adaptación en las relaciones laborales generadas de la organización; los individuos desde el desempeño laboral contribuyen con el clima organizacional, de modo que aunque haya un compromiso individual, estos convergen en fines colectivos, aprenden para que la organización aprenda y ponen al servicio de la institución su experiencia y conocimiento. 


\section{Conclusiones}

De lo que se trata es de conversar con los otros, a fin de, comprendiéndolos, ampliar el universo del discurso humano. Clifford Geertz

La organización objeto de estudio es una institución de educación superior, enmarcada en la filosofía de la economía solidaria. Los individuos vinculados laboralmente profesan estos mismos principios pues una vez ingresan, los capacitan en la filosofía institucional. La organización asume el recurso humano como el elemento relevante que expone la fuerza de trabajo y se enfoca en el logro de los propósitos y fines organizacionales. A partir de una mirada antropológica y de la administración sobre la cultura organizacional y su implementación, las relaciones laborales son determinantes para lograr mejor conocimiento de las caracterizaciones socioculturales que inciden en el rendimiento productivo y competitivo de la organización.

Comprender la interculturalidad como la relación entre dos o más individuos lleva a la comprensión y valoración del otro; el talento humano es un factor relevante en las organizaciones y con su ingenio y creatividad transfiere conocimiento para dinamizar la gestión e impulsar el desarrollo y crecimiento de estas. No puede haber organizaciones sociales sin la participación de los individuos y en esta relación se fortalece la capacidad de trabajo en función de la institución.

Las organizaciones como unidad social, en la que aprenden los unos de los otros, se identifican con valores comunes, se recrean e intercambian ideas, formas de pensar y actitudes; todo esto permite el crecimiento personal y social de las comunidades. Se considera que el hombre es incapaz de vivir fuera de las organizaciones, porque por naturaleza es un ser social y en este escenario le es posible encontrarse consigo mismo y con el otro, en busca de una vida plena y de bienestar.

Los individuos son seres sociales que se acomodan a diversos ambientes; de su grado de adaptación dependen la satisfacción y la motivación para el desarrollo de las actividades laborales en función de la productividad. La actitud, los principios, los valores y la influencia del proceso de socialización contribuyen a fortalecer las competencias para lograr el mejoramiento continuo y alcanzar desempeños efectivos en la vida laboral y en las relaciones en las que está inmerso el individuo. El grupo en general manifiesta empatía y disposición de adaptación a las condiciones que propicia la organización para el desarrollo del trabajo.

Explicar los imaginarios de los diferentes grupos con respecto a las funciones y roles que desempeñan los individuos vinculados laboralmente: la organización es una sola y sus integrantes tienen diversas miradas acordes con el estilo de vida, la cultura, el nivel socioeconómico, la formación académica, la edad, el género y estratificación social. Los imaginarios de las relaciones se establecen en las estructuras empresariales, la vida en el contexto cultural y la aceptación de las diferencias de la vida cotidiana laboral. La administración propicia ambientes para el desempeño de los individuos y la contabilidad social regula la dinámica organizacional a partir de la satisfacción percibida por el capital humano. 
Finalmente, se deja abierta la posibilidad de profundizar en la temática sobre la función y el aporte de la antropología sociocultural, la administración y la contabilidad social en las organizaciones pues la incursión desde el enfoque teórico práctico es reciente. El ser humano tiene un alto grado de participación en la sostenibilidad organizacional; por esto, las líneas de interés que se establecen para generar nuevos proyectos se orientan a contabilidad social, sociedad y ambiente; productividad, competitividad y clima de negocios; cultura organizacional y responsabilidad social empresarial.

\section{Referencias}

Arbaiza-Fermini, Lydia Aurora (2011). Alianzas estratégicas: instrumento de negociación y desarrollo sostenible mirado desde la perspectiva de la interculturalidad. Revista EAN, 70, 102-117. Disponible en: http:// journal.ean.edu.co/index.php/Revista/ article/view/537/526

Cáceres-Rubio, Felipe \& Villacrés-Chaparro, Mónica (2010). Cultura organizacional y las fusiones empresariales. Facultad de Psicología, Universidad de La Sabana. Disponible en: http://intellectum.unisabana.edu. co/bitstream/handle/10818/4045/131294. pdf? sequence $=1$

Castoriadis, Cornelius $(1975,1998)$. The Imaginary Institution of Society. Cambridge, Massachusetts: The MIT Press.

Castoriadis, Cornelius (1997). El imaginario social instituyente. Zona Erógena, 35, 1-9. Disponible en: http://datateca.unad.edu. co/contenidos/100001/100001_2014_II/
Castoriadis_Cornelius_-_El_Imaginario_ Social_Instituyente.pdf

Chanlat, Jean François (1994). Hacia una antropología de la organización. Gestión y Política Pública, 3 (2), 317-364. Disponible en: http://www.gestionypoliticapublica. cide.edu/num_anteriores/Vol.III_ No.II_2dosem/CJ_Vol.III_No.II_2sem.pdf Chua, Wai Fong (1986). Radical Developments in Accounting Thought. The Accounting Review, 61 (4), 601-632.

García de la Torre, Consuelo (2007). Estudios sobre identidad y cultura en las organizaciones en América Latina. Cuadernos de Administración, 38, 21-51. Disponible en: http://bibliotecadigital.univalle.edu.co/ bitstream/10893/2151/1/ESTUDIOS\%20 SOBRE\%20LA\%20IDENTIDAD\%20Y\%20

\section{LA\%20CULTURA.pdf}

Gauthey, Franck; Ratiu, Indrei; Rodgers, Irène $\&$ Xardel, Dominique (1988). Leaders sans frontières: Le défi des différences. Auckland: McGraw-Hill.

Geertz, Clifford (1992). Los usos de la diversidad. Barcelona: Paidós.

Geertz, Clifford (2003). La interpretación de las culturas. Barcelona: Gedisa.

Gonnet, Juan Pablo (2012). Cultura, organizaciones y antropología. Una revisión crítica. Avá (Posadas), Revista de Antropología, 21, 1-20. Disponible en: http:// www.scielo.org.ar/pdf/ava/n21/n21a07. pdf, http://www.redalyc.org/articulo. oa?id=169030268008

Guber, Rosana (2000). La etnografía. Método, campo y reflexividad. Bogotá: Grupo Editorial Norma. Disponible en: 
UNA PERSPECTIVA ANTROPOLóGica de LAS ORGANIZACIONes / C. CELY, o. Gómez / 199

https://www.academia.edu/296632/La_ Etnograf\%C3\%ADa

Jiménez-Aguirre, Rubiela (2008). Reflexiones sobre la teoría de la responsabilidad social empresarial: una mirada desde la contabilidad. En Perspectivas críticas de la Contabilidad, Reflexiones y críticas contables alternas al pensamiento único, Memorias VII Simposio Nacional de Investigación Contable y Docencia, 213-225. Bogotá: Universidad Nacional de Colombia.

Kottak, Conrad Phillip (2011). Antropología cultural. México: McGrawHill.

Machado-Rivera, Marco Antonio (2007). La responsabilidad social corporativa como resultante de un cambio en los imaginarios del contador público: del mecanicismo a la complejidad de las organizaciones. Contaduría Universidad de Antioquia, 51, 6787. Disponible en: http://aprendeenlinea. udea.edu.co/revistas/index.php/cont/article/ viewFile/2148/1750

Macías, Hugo A. (2013). Vínculos de la investigación interpretativa con la producción académica colombiana: avances y oportunidades. Cuadernos de Contabilidad Universidad Javeriana, 14 (35), 699-727. Disponible en: http:// cuadernosdecontabilidad.javeriana.edu.co/ vol14_n_35/vol14_35_12.pdf

Martínez-Miguélez, Miguel (2000). La investigación cualitativa etnográfica en educación. México: Editorial Trillas.

Morgan, Gareth (1998). Imágenes de la organización. México: Alfaomega Grupo Editor. Ocampo-Salazar, Carmen Alejandra; LastraRodríguez, Catherin \& Gómez-Martínez,
Juan Carlos (2008). Algunas relaciones entre contabilidad y cultura. Contaduría Universidad de Antioquia, 52, 215-258. Disponible en: http://aprendeenlinea.udea. edu.co/revistas/index.php/cont/article/ view/2170/1764

Ospina-Zapata, Carlos Mario (2006). Las tramas de la contabilidad: trazos para quienes empiezan su formación en contaduría pública. Contaduría Universidad de Antioquia, 48, 155-186. Disponible en: https://aprendeenlinea.udea.edu. co/revistas/index.php/cont/article/ view/25694/21215

Pfeffer, Jeffrey (2000). Nuevos rumbos en las teorías de la organización. Problemas y posibilidades. María del Pilar Carril-Villarreal, trad., México: Oxford University Press.

Rey, Françoise (1978). Introduction à la comptabilité sociale: domaines, techniques et applications. Paris: Entreprise Moderne d'Edition.

Ritzer, George (2002). Teoría sociológica moderna. Madrid: McGraw-Hill.

Sandoval-Casilimas, Carlos A. (1996). Investigación cualitativa. Bogotá: Instituto Colombiano para el Fomento de la Educación Superior, ICFES. Disponible en: http://panel.inkuba.com/sites/2/archivos/ manual\%20colombia\%20cualitativo.pdf

Schein, Edgar H. (1988). La cultura empresarial y el liderazgo. Una visión dinámica. Barcelona: Plaza y Janés Editores.

Sunder, Shyam (2005). Teoría de la contabilidad y el control. Bogotá: Universidad Nacional. Disponible en: http://faculty.som.yale.edu/ shyamsunder/Research/Accounting\%20 
200 / VOL. 17 / NO. 43 / ENERO-JUNIO 2016

and\%20Control/Books/Teoria_de_la_ contabilidad_2015.pdf

Wright, Susan (ed.) (2005). Anthropology of Organizations. London: Routledge.

- Fecha de recepción: 3 de febrero de 2016

- Fecha de aceptación: 17 de junio de 2016

- Disponible en línea: 30 de junio de 2016

\section{Para citar este artículo}

Cely-López, Carmen Luz \& Gómez-Niño, Ofelia (2016). Una perspectiva antropológica de las organizaciones desde la caracterización sociocultural, administración y contabilidad social. Caso institución de educación superior. Cuadernos de Contabilidad, 17(43), 183-201. http://dx.doi. org/10.11144/Javeriana.cc17-43.paoc 
UNA PERSPECTIVA ANTROPOLÓGICA DE LAS ORGANIZACIONES / C. CELY, O. GÓMEZ / 201

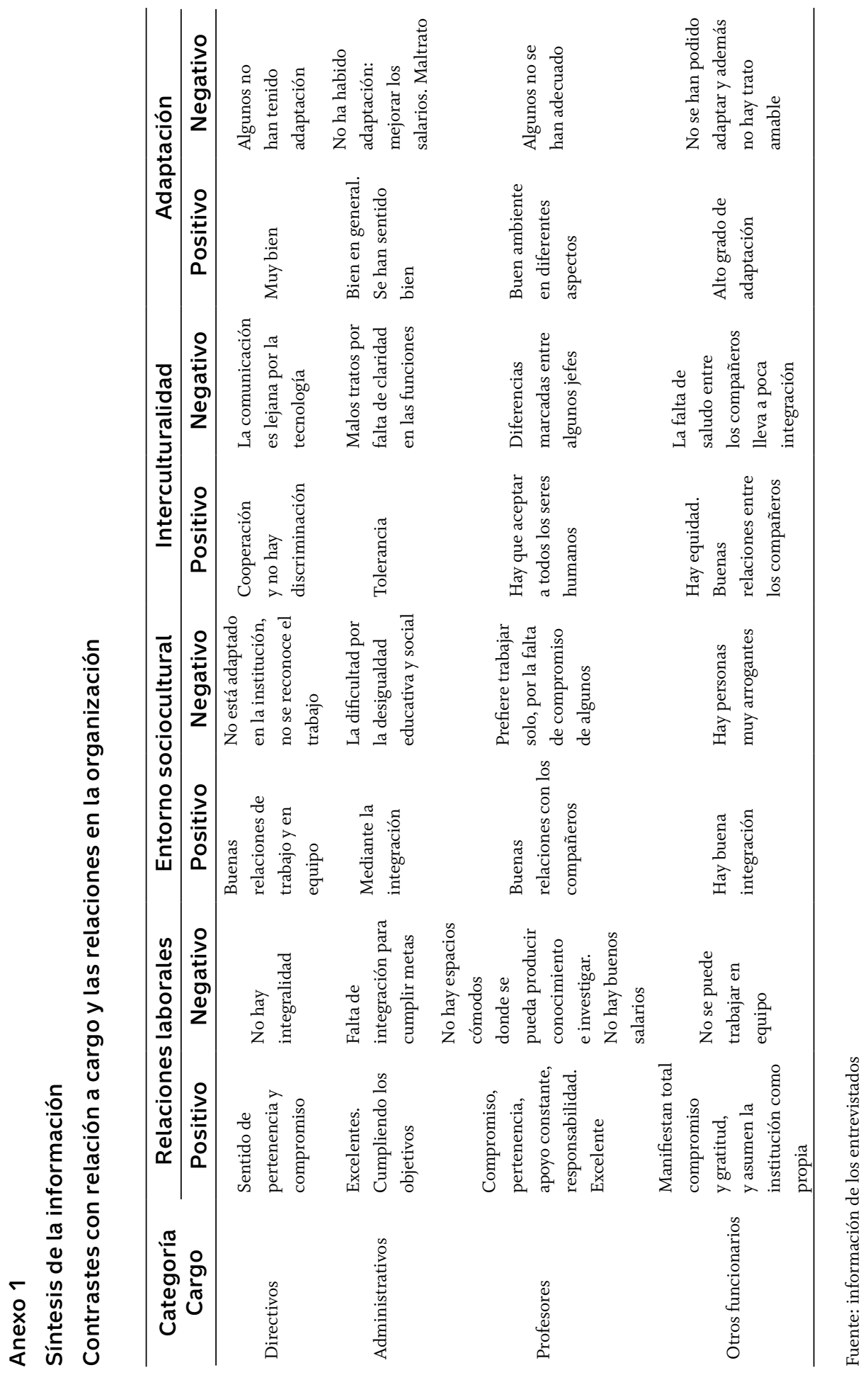


